Published in Fluct. Noise Lett. 3, L91-L95 (2003).

\title{
MID-INFRARED LASING INDUCED BY NOISE
}

\author{
V. V. SHERSTNEV*, A. KRIER, A. G. BALANOV, N. B. JANSON, A. N. SILCHENKO \\ and P. V. E. McCLINTOCK \\ Department of Physics, University of Lancaster, LA1 $4 Y B, U K$ \\ *v.sherstnev@lancaster.ac.uk \\ Received (19 December 2002) \\ Revised (6 February 2003) \\ Accepted (21 February 2003)
}

\begin{abstract}
We demonstrate that external noise can play a constructive role in a laser diode, inducing coherent mid-infrared radiation. Depending on noise intensity, the induced lasing can be either unimode or mulitimode. The coherence of the radiation in each mode reaches its maximum at an optimal noise intensity that differs depending on the mode. The phenomenon can therefore be classified as a multiple coherence resonance.
\end{abstract}

Keywords: Noise pumping; infrared lasing; noise-induced ordering; coherence resonance.

\section{Introduction}

Infrared emitting laser diodes (LDs) have a growing number of applications. In practice, lasing is normally induced by coherent energy sources e.g. optical pumping by coherent radiation or by an electrical current, with the latter being either constant or pulsed [1-4]. It is well known that noise is inevitably present in all semiconducting devices, including lasers, and it is widely acknowledged that it normally plays a negative role. In addition to thermal fluctuations, random fluctuations in the pump also contribute to the noise. They usually lead to a deterioration of the laser: the intensity of the radiation decreases and the peak in the spectrum broadens. For these reasons, the idea of utilizing noise in a semiconductor device to produce any kind of positive effect appears at first sight totally unrealistic.

In this Letter we report, however, that external noise can play a constructive role in a laser diode, causing effects that are both nontrivial and counter-intuitive. We find that the device is able to transform the energy of the random pump fluctuations into coherent mid-infrared radiation. Noise-induced lasing can be either single- or multi-mode, each mode attaining its maximum coherence at an optimal noise level. The results reported allow one to reconsider the role of random fluctuations in semiconductors, and might lead to a new direction in their study. 


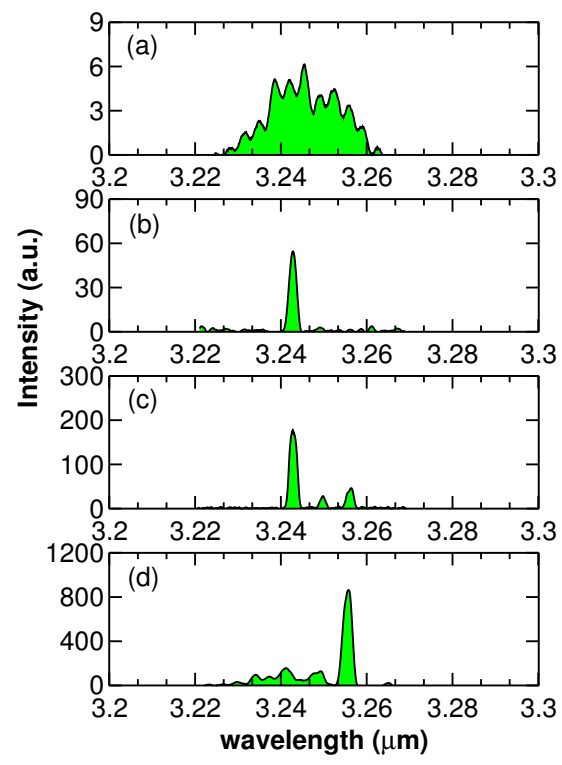

Fig 1. Electroluminescence spectra obtained for different values of noise pumping: a) $4.1 \mathrm{~mA}$, b) $4.46 \mathrm{~mA}, \quad$ c) $5.35 \mathrm{~mA}$, d) $11.59 \mathrm{~mA}$.

\section{Semiconducter Diode Under Study}

The semiconductor laser chosen for our investigation consists of a double heterostructure $(\mathrm{DH})$, which provides an optical waveguide, an active region, and two electrical contacts. We used an LD fabricated from III-V double heterostructures (DHs) grown by liquid phase epitaxy (LPE). A conventional horizontal, multi-well graphite sliding boat was used for the LPE growth of the LD structures onto ptype InAs (100) substrates with a carrier concentration of $10^{18} \mathrm{~cm}^{-3}$ obtained from Wafer Technology Ltd. The resulting epitaxial structure, which has been described in detail elsewhere [5], was a DH in which the unintentionally doped n$\mathrm{InAs}_{0.94} \mathrm{Sb}_{0.06}$ active layer is enclosed between $\mathrm{P}-$ and $\mathrm{N}-\mathrm{InAs}_{0.42} \mathrm{Sb}_{0.18} \mathrm{P}_{0.40}$ confinement layers. The $\mathrm{P}$ content in the confinement layers was $0.40\left(E_{g}=620 \mathrm{meV}\right)$ to provide high bandgap energy and large interface band offsets for good carrier confinement. The $\mathrm{InAs}_{0.94} \mathrm{Sb}_{0.06}$ active region was $0.7 \mu \mathrm{m}$ thick $\left(E_{g}=350 \mathrm{meV}\right)$, and the $\mathrm{InAs}_{0.42} \mathrm{Sb}_{0.18} \mathrm{P}_{0.40}$ layers, each being $3.0 \mu \mathrm{m}$ in thickness. Residual carrier concentration in the active layer was reduced to $10^{16} \mathrm{~cm}^{-3}$. The cladding layers were intentionally doped with Sn up to a concentration of $5 \cdot 10^{18} \mathrm{~cm}^{-3}$ and with Zn up to $10^{18} \mathrm{~cm}^{-3}$ for $\mathrm{N}$ - and $\mathrm{P}$-sides, respectively. The LDs were fabricated from the epitaxial wafers using standard procedures. Ohmic contacts were formed by thermal evaporation of Au:Zn and Au:Te alloys on the $\mathrm{p}-$ and $\mathrm{n}$-sides of the structures, respectively. Double-channel mesa-stripe laser chips with a $b=12 \mu \mathrm{m}$ stripe width were then processed using photolithography. Laser diodes were cleaved with a Fabry-Perot cavity length $(L)$ of $300 \mu \mathrm{m}$. The LD chips were then mounted p-side up onto TO-49 headers for testing. The laser was placed in a metal dewar and cooled to $77 \mathrm{~K}$. After passing through the cryostat windows, the radiation was 

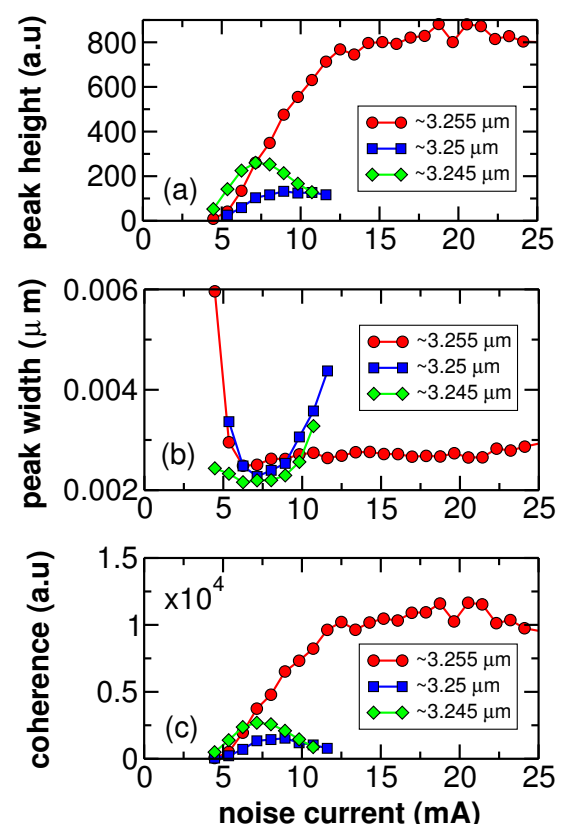

Fig 2. Dependence of the mode coherence on noise current. The different symbols correspond to the different optical emission modes, as indicated.

collected using $\mathrm{CaF}_{2}$ lenses and focused into a $1 \mathrm{~m}$ Monospek 1000 monochromator. The electroluminescence was detected using a cooled $(77 \mathrm{~K}) \mathrm{InSb}$ photodiode detector and Stanford Research (SR850) digital phase-sensitive detector. Normally, pumping the laser with conventional rectangular pulses of current of amplitude above the generation threshold $29 \mathrm{~mA}$ (at a repetition frequency of $10 \mathrm{kHz}$ ), results in single-mode laser emission at $3.3 \mu \mathrm{m}$.

\section{Experimental Results and Discussion}

In our experiments, instead of the usual pumping in the form of a sequence of unipolar pulses, a random signal with zero mean was applied, having a Gaussian distribution and spectral bandwidth $>100 \mathrm{kHz}$ [6]. Figure 1 shows the spectral structure of the electroluminescence for different values of noise current. For a root mean square (rms) noise current of $4.1 \mathrm{~mA}$ only spontaneous emission was observed (Fig. 1(a)). However, with further increase of the noise intensity, a remarkable spectral narrowing took place (Fig. 1(b)), quite contrary to the traditional expectation. This narrowing was accompanied by a substantial increase of optical emission power and thus can be considered as the beginning of laser emission. Further increase of noise intensity led to the growth of radiation intensity. Above 4.5 $\mathrm{mA}$, two additional modes appeared sequentially in the optical emission (Fig. 1(c)). As the noise intensity was increased, the spectral peak associated with each particular mode grew to reach its maximum height and minimum width at a (different) optimal noise level.

With further increase of noise, the peaks' heights decreased while their widths 
grew monotonically. The lasing modes were gradually destroyed, starting with that of smallest wavelength (Fig. 1(d)). In Fig. 2(a,b) the dependences of height (a) and width (b) on noise intensity for the three most pronounced modes are shown. It is clearly seen that, for all peaks considered, these dependences are nonmonotonic, i.e. the coherence of the radiation is changed by variation of the noise intensity. To estimate the coherence of each radiation mode, we use a parameter that can serve as an analogue of the signal-to-noise ratio [7-11] widely used in oscillation theory to describe spectral broadening due to noise: coherence $=$ height $\times$ wavelength $/$ width . Here, "height" is the height of the peak, "width" is its width at the half-height, and "wavelength" is its centre wavelength. Fig. 2(c) shows the coherence of each lasing mode as a function of the rms noise current. The optimal noise intensity, providing the maximum coherence of the noise-induced radiation mode, is unique for each peak. Thus, by choosing different noise intensities, one is able to suppress or enhance the coherence of a selected mode. It is interesting to note that, by exciting particular modes, the noise in our laser diode effectively reveals the inner structure of the quantum energy levels, thus constituting another example of the creative role of noise.

The observed effects can be accounted for in terms of nonlinear dynamics. The laser can be viewed as a threshold device that starts to generate a periodic signal when the input signal exceeds this threshold, i.e. it is an excitable system. Thus, if noise is applied to it, signal generation will occur and disappear at random times [12]. When the noise is weak, the fraction of the observation time when oscillations occur is small, and the spectrum of the total signal at the output is broadband continuous with small energy. As the noise becomes stronger, oscillatory modes are excited more frequently, the output energy grows and the spectrum narrows. It is clear, however, that for very large noise the oscillations in the device will become very irregular again. In between, there must exist some optimal noise intensity for which the total output of the system is maximally coherent. Thus, the effects observed in this work can be accounted for in terms of the recently identified coherence resonance phenomenon $[13,14]$. Its characteristic signature is a bellshaped dependence of the signal-to-noise ratio or equivalent characteristic on the noise intensity. However, the effects observed here, although similar, are actually more complex. In our case, coherence resonance occurs separately for each mode of optical emission, i.e. each mode acquires its maximum coherence at a different noise intensity. It therefore seems appropriate to call the phenomenon multiple coherence resonance.

\section{Conclusions}

We have shown that pumping by noise alone is able to induce multimode lasing in a semiconducter diode. The spectral characteristics of this lasing exhibit an interesting dependence on noise intensity. For each mode there are certain values of noise intensity at which the corresponding spectral peak has maximal height and minimal width, indicative of noise-induced ordering. In fact, variation of the applied noise intensity allows one to control the coherence of a particular mode. This property looks promising for application to problems in e.g. telecommunications.

The observed phenomena appear to be closely related to coherence resonance. 
The results obtained compel us to take a new view of the role of noise in semiconductors, and probably provide a way to utilize noise energy for laser radiation.

\section{Acknowledgements}

The research has been supported by the Engineering and Physical Sciences Research Council (UK) and by the Leverhulme Trust (UK).

\section{References}

[1] E H. Aifer, W. W. Bewley, C. L. Felix, I. Vurgaftman, L. J. Olafsen, J. R. Meyer, H. Lee, R. U. Martinelli, J. C. Connolly, A. R. Sugg and G. Olsen, CW operation of $3.4 \mu m$ optically-pumped type-II W laser to 220K, Electronic Letters 34 (1998) 1587-1588.

[2] I. Vurgaftman, C. L. Felix, W. W. Bewley, D. W. Stokes, R. E. Bartolo, J. R. Meyer and K. L. Clark, Mid-infrared 'W' lasers, Phil. Trans. Roy. Soc. Series A 359 (2001) 489-503.

[3] J. C. Nicolas, A. N. Baranov, Y. Cuminal, Y. Rouillard and C. Alibert, Tunable diode

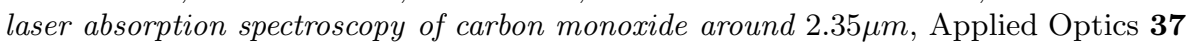
(1998) 7906-7911.

[4] H. Lee, L. J. Olafsen, R. J. Menna, W. W. Bewley, R. U. Martinelli, I. Vurgaftman, D. Z. Garbuzov, C. L. Felix, M. Maiorov, J. R. Meyer, J. C. Connolly, A. R. Sugg and G. H. Olsen, Room-temperature type-II $W$ quantum well diode laser with broadened waveguide emitting at $\lambda=3.30 \mu m$, Electronic Letters 35 (1999) 1743-1745.

[5] S. Civis, A. N. Imenkov, A. P. Danilova, N. M. Kolchanova, V. V. Sherstnev, Y. P. Yakovlev and A. D. Walters, Tunable single-mode $3.2 \mu m$ laser based on InAsSb/InAsSbP double heterostructure with drive-current tuning range of $10 \mathrm{~cm}^{-1}$, Spectrochimica Acta Part A: Molecular and Biomolecular Spectroscopy 56 (2000) $2125-2130$

[6] D. G. Luchinsky, P. V. E. McClintock and M. I. Dykman, Analogue studies of nonlinear systems, Rep. Prog. Phys. 61 (1998) 889-997.

[7] K. Wiesenfeld and F. Moss, Stochastic resonance and the benefits of noise: from ice ages to crayfish and SQUIDs, Nature 373 (1995) 33-36.

[8] M. I. Dykman and P. V. E. McClintock, What can stochastic resonance do?, Nature 391 (1998) 344.

[9] L. Gammaitoni, P. Hänggi, P. Jung and F. Marchesoni, Stochastic resonance, Rev. Mod. Phys. 70 (1998) 223-285.

[10] V. S. Anishchenko, A. Neiman, F. Moss and L. Schimantsky-Geier, Stochastic resonance: noise induced order, Usp. Fiz. Nauk 169 (1999) 7-36, [Sov. Phys. Usp. 42 (1999) 7-36].

[11] M I Dykman, D G Luchinsky, R Mannella, P V E McClintock, N D Stein and N G Stocks, Stochastic resonance in perspective, Nuovo Cimento D 17 (1995) 661-683.

[12] P.J. Edwards and R.B. Hurst, Level-crossing statistics of the horizontal wind speed in the planetary surface boundary layer, CHAOS 11(3) (2001) 611-618.

[13] H. Gang, T. Ditzinger, C. Z. Ning and H. Haken, Stochastic resonance without external periodic force, Phys. Rev. Lett. 71 (1993) 807-810.

[14] A. S. Pikovsky and J. Kurths, Coherence resonance, Phys. Rev. Lett. 78 (1997) 775778. 